\title{
Distribution, caractérisation écologique de l'habitat et notes sur le régime alimentaire du phacochère commun, Phacochoerus africanus (Gmelin, 1788) au Sud du Bénin
}

\author{
Christophe K. ADJIN ${ }^{1}$, Toussaint O. LOUGBEGNON ${ }^{1,3^{*}}$, Jean T. Claude CODJIA ${ }^{1}$ \\ et Guy Apollinaire MENSAH ${ }^{2}$ \\ ${ }^{1}$ Laboratoire Aménagement des Forêts et de Biogéographie (LAF-Bio), Faculté des Sciences agronomiques, \\ Université d'Abomey-calavi. \\ ${ }^{2}$ Maître de recherche, Directeur du CRA-Agonkanmey/Institut National de la Recherche Agronomique du \\ Bénin. \\ ${ }^{3}$ Ecole Nationale Supérieure des Sciences et Techniques Agronomiques (ENSTA-Kétou), Université d'Abomey- \\ Calavi (UAC), Bénin. \\ *Auteur correspondant, E-mail : tlougbe@yahoo.fr
}

\section{RESUME}

Phacochoerus africanus est le seul suidae du genre Phacochoerus présent au Bénin, souvent rencontré en zones soudaniennes et soudano-guinéennes (Nord et Centre). Cette étude fait état de la présence effective de l'espèce dans la partie guinéenne du Bénin et fait ressortir quelques données préliminaires sur sa distribution, la caractérisation de son habitat et son régime alimentaire. L'habitat a été étudié sur la base d'observations directes et des indices de présence, tandis que les entretiens avec 42 chasseurs complétés avec des observations directes de restes d'aliments et d'analyses macroscopiques de crottes ont permis d'appréhender son régime alimentaire. Sur l'ensemble des deux forêts prospectées, Phacochoerus africanus se retrouve essentiellement dans la forêt classée de Dogo-Kétou. Il vit dans des gîtes, constitués d'espèces végétales de hauteur moyenne $1,95 \pm 0,49 \mathrm{~m}$ et de circonférence 16,2 $\pm 4,58 \mathrm{~m}$. Il fréquente les forêts claires arbustives à Pterocarpus erinaceus et Anogeissus leiocarpus de recouvrements compris entre 0 et 50\%. Dans ces habitats, les sols sont argileux et limoneux avec la présence de cours d'eau et de marigots temporaires qui lui servent de bauges. Son régime alimentaire est constitué de feuilles, d'inflorescences, de fruits, de tiges, de petits mammifères, de poissons et les vers de terre.

(C) 2011 International Formulae Group. All rights reserved.

Mots clés : Phacochoerus africanus, écologie, habitat, régime alimentaire, sud du Bénin.

\section{INTRODUCTION}

Deux espèces de phacochères sont présentes sur le continent africain, à savoir Phacochoerus aethiopicus (Pallas, 1766) et Phacochoerus africanus (Gmelin, 1788). Le phacochère commun, $P$. africanus se différencie de la première par la présence d'incisives fonctionnelles sur la mâchoire supérieure, un crâne relativement plus long mais moins large et une taille moyennement plus grande (Grubb, 1993). Phacochoerus africanus est présente sur une large aire de répartition s'étendant des régions de savanes et sahéliennes du Sénégal jusque dans les steppes arbustives et les broussailles à feuilles persistantes des régions montagneuses de 
Golis et Wagon en Somalie du Nord (Vercammen et Mason, 1993; D'Huart et Grubb, 2001). On la retrouve également dans l'inhospitalier désert de Danakil en Erythrée, sur les terres arides de savane ou légèrement boisées de l'Ethiopie en dessous des $2000 \mathrm{~m}$ d'altitude (D'Huart et Grubb, 2001). Les phacochères communs vivent aussi dans la partie Sud de l'Afrique (Vercammen et Mason, 1993). Ils présenteraient provisoirement selon Grubb (1993), quatre sous-espèces notamment $P$. a. africanus, $P$. $a$. aelini, $P$. a. massaicus et $P$. a. sundevalli.

Au Bénin, $P$. africanus est le seul de ce genre rencontré dans les savanes et forêts claires des zones phytogéographiques soudaniennes ou guineo-soudaniennes (Miade, 1998; Sinsin et al., 1998 ; Agassounon, 2005 ; Codjia et al., 2007). Espèce très braconnée, elle sert de ressources alimentaires et de sources de revenus pour les populations locales riveraines des aires protégées (Sinsin et al., 1997a). Le phacochère commun a fait l'objet de peu d'études au Bénin (Miade, 1998 ; Agassounon, 2005 ; Codjia et al., 2007) et a été identifié dans plusieurs dénombrements fauniques (Sinsin et al., 1997b ; Sinsin et al., 1998). La latitude la plus basse de son aire de distribution au Bénin a été définie comme étant celle qui couvre la région des Monts Kouffé, localisée au centre du Bénin entre $8^{\circ}$ et $9^{\circ}$ de latitude Nord et entre $1^{\circ} 50$ ' et $2^{\circ} 30^{\prime}$ de longitude Est, dans la zone phytogéographique de transition soudanoguinéenne, au dessus du Dahomey Gap (Sinsin et al., 1998).

Ce travail présente les observations récentes de l'espèce faites au sud du Bénin dans une zone phytogéographique à affinités guinéo-congolaises et retrace sa distribution écologique ainsi que les données préliminaires liées à son régime alimentaire.

\section{MATERIEL ET METHODES \\ Milieu d'étude Situation géographique}

Les sites d'étude sont situés au sud du Bénin. Ils constituent deux entités principales : la forêt classée de Dogo-Kétou et la forêt marécageuse qui entoure le lac Azili (Figures 1 et 2). L'aire d'étude s'étend entre les latitudes $7^{\circ} 14^{\prime}$ et $7^{\circ} 40^{\prime} \mathrm{N}$ et les longitudes $2^{\circ} 22^{\prime}$ et $2^{\circ} 40^{\prime} \mathrm{E}$ et couvre une superficie totale 48210 ha. La forêt de Dogo-Kétou est une formation de savane soudano-guinéenne. Quant à la forêt du lac Azili, c'est une formation marécageuse de type guinéen. Ce complexe d'écosystèmes terrestre et marécageux contigus est très intéressant pour servir de gamme d'habitats aux phacochères.

\section{Climat}

Le climat de la région d'étude est de type sub-équatorial à deux saisons pluvieuses (avril-juin et septembre-octobre) avec une pluviométrie moyenne annuelle de $1012 \mathrm{~mm}$. La température moyenne annuelle est de 27,7 ${ }^{\circ} \mathrm{C}$. L'humidité relative moyenne s'abaisse jusqu'à $37{ }^{\circ} \mathrm{C}$ pendant la saison sèche. L'insolation moyenne annuelle est de 2135 heures et l'évapotranspiration potentielle (ETP) est de 1504,7 mm/an en moyenne (Tohinlo, 2001). C'est cette ambiance climatique qui héberge la faune dans cette partie du Bénin.

Végétation

La zone d'étude est caractérisée par un ensemble de formations éco-floristiques varié notamment de galeries forestières à Pterocarpus santalinoides, Cola laurifolia, Cynometra megalophylla et Diospyros mespiliformis; de forêts claires à Anogeissus leiocarpus, Combretum collinum, Isoberlinia doka et Pterocarpus erinaceus; de savanes boisées à Vitellaria paradoxa et Combretum collinum; de savanes arborées / arbustives à Combretum collinum et Lophira lanceolata, d'un ensemble de groupements post-culturaux que sont les mosaïques de champs et jachères à Brachiaria deflexa et Flueggea virosa et d'un ensemble de forêts marécageuses à Raphia hookeri, Pterocarpus santalinoides et Nauclea xanthoxylon (Bossou, 2001 ; Tohinlo, 2001).

\section{Sols et réseau hydrographique}

La région d'étude est caractérisée par une grande hétérogénéité de sols. Les plus importants sont les sols sur colluvions et 
alluvions, limoneux-sableux sur argile, sableuse à hydromorphie temporaire qui dominent dans la forêt classée de Dogo-Kétou et les sols homogènes sablo-argileux profonds sur lesquels repose la forêt d'Azili (Bossou, 2001 ; Tohinlo, 2001). La rivière Issaoun, le fleuve Ouémé et le lac Azili constituent l'essentiel de l'hydrographie.

\section{Faune mammalienne}

Les mammifères les plus fréquents sont le céphalophe à flanc roux (Cephalophus rufilatus), le phacochère (Phacochoerus africanus), le potamochère (Potamochoerus porcus), le guib harnaché (Tragelaphus scriptus), le sitatunga (Tragelaphus spekei), le cobe de Buffon (Kobus kob), le lièvre (Lepus saxatilis), l'aulacode (Thryonomys swinderianus), l'écureuil fouisseur (Xerus erythropus) et les porcs-épic (Hystrix cristata et Atherurus africanus) (Bossou, 2001).

\section{Méthodes d'étude \\ Recherche des paramètres de distribution des phacochères}

Elle est faite au moyen des prospections pédestres directes dans les zones préalablement identifiées en compagnie de chasseurs locaux. Ces prospections sont faites suivant des transects avec des azimuts bien déterminés en tenant compte de certains facteurs tels que la direction du vent, les aspects de la végétation et de la géomorphologie (Figures 3 et 4). Au total, 51 transects avec aller simple, de plus de $7 \mathrm{~km}$ de longueur chacun, ont étés parcourus en trois mois (90 jours) sur une superficie totale de 1693 ha. Ces prospections ont été effectuées pendant la saison pluvieuse (juillet à septembre) qui est la période de pleine et d'intense activité de l'animal. Compte tenu des mœurs diurnes des phacochères, elles commencent dans la matinée à 7 heures et se terminent dans l'après-midi à 16 heures. Lors des prospections, les objectifs poursuivis sont surtout l'observation des animaux et ou la détection de leurs indices de présence (les gîtes, les empreintes, les crottes et les fouilles).
Le gîte se présente sous forme de dôme et est constitué en fourrée dense faite de buissons et de lianes entremêlés avec un intérieur sec (photo 1). Les fouilles sont la principale activité des phacochères durant la journée. Et les coups de boutoirs sont bien visibles après leurs passages (de la Fuente, 1979).

Ensuite, les coordonnées géographiques des lieux de contact avec les animaux et les indices de présence sont prises au GPS.

\section{Recherche des éléments de caractérisation de l'habitat des phacochères}

Une fois un gîte repéré, sa circonférence et sa hauteur sont mesurées. Ensuite, on passe à l'identification des espèces végétales qui le constituent et à la description de l'habitat du phacochère. Cette description comprend la détermination du type de végétation, de son recouvrement, de sa structure verticale et de sa composition floristique. Les caractéristiques du sol et des sources d'eau environnantes sont également décrites.

\section{Analyse du régime alimentaire des phacochères}

Les entretiens avec 42 chasseurs couplés avec des observations directes de restes d'aliments et d'analyses macroscopiques de 8 tas de crottes ont permis d'appréhender le régime alimentaire. La reconnaissance du régime alimentaire à partir des restes d'aliment du phacochère s'est faite en collaboration avec le degré de connaissance des chasseurs.

\section{Analyses des données collectées}

L'ensemble des coordonnées GPS enregistrées sur la distribution de l'espèce ont été exportées dans le logiciel Arcview. Ce qui a permis de réaliser la carte de distribution de l'espèce dans les habitats en présence.

Des calculs de moyennes arithmétiques et d'écart-types ont été faits sur les éléments de caractérisation de l'habitat (hauteur et circonférence). Elles ont permis de préciser les tendances des dimensions des gîtes.

La moyenne $x$ s'écrit : 
$x=\frac{x 1+x 2+\cdots x i+\cdots x n}{n}$ où xi représente

les hauteurs et les circonférences des ni gîtes et $\mathrm{n}$ est la somme totale des hauteurs et des circonférences des gîtes.

L'écart type s'écrit : $\sigma_{X}=\sqrt{S^{2}}$ où $S$ est la variance.

La formule de la fréquence est: $\boldsymbol{F}=\frac{\boldsymbol{n} \boldsymbol{i}}{\boldsymbol{n}}$ où ni représente la fréquence élémentaire du facteur considéré et $\mathrm{n}$ est la proportion totale de la modalité du facteur considéré.

Les données relatives à la description de l'habitat ont été analysées à base de tableaux de fréquence. Pour ce qui est de régime alimentaire, des calculs de proportions des divers items consommés ont étés effectués.

\section{RESULTATS}

Habitat des phacochères : distribution des phacochères à travers les deux complexes de forêts

Les prospections faites sur l'ensemble des deux écosystèmes ont révélées que les phacochères se retrouvent uniquement dans la forêt classée de Dogo-Kétou et les milieux connexes. Aucune trace de l'espèce n'est retrouvée curieusement dans les forêts marécageuses du lac Azili. A Dogo-Kétou, un total de huit (8) gîtes a été repéré (Figure 5).

\section{Description des gîtes}

Les gîtes présentent un aspect de dôme formé de plusieurs espèces végétales. Cellesci sont constituées soit des arbustes d'Anogeissus leiocarpus, de Flueggea_virosa ou de Zanthoxylum zanthoxyloides qui servent de supports aux fourrées de Chromolaena odorata et dans les lianes de Cissus spp. La hauteur des gîtes varie entre $1,2 \mathrm{~m}$ et $2,7 \mathrm{~m}$ avec une moyenne de 1,95 $\pm 0,45 \mathrm{~m}$, tandis que la circonférence externe moyenne est de $16,2 \pm 4,58 \mathrm{~m}$ avec un maximum de $22,8 \mathrm{~m}$ et un minimum de $9,5 \mathrm{~m}$. Les gîtes ont une entrée unique située à ras le sol et légèrement sur creusée (Tableau 1).

\section{Analyse des éléments de caractérisation de l'habitat des phacochères}

Les phacochères dans cette partie du Sud-Bénin fréquentent surtout les savanes arborées et arbustives (65\%) à Pterocarpus erinaceus et Anogeissus leiocarpus ; les forêts claires $(25 \%)$ à Vitellaria paradoxa, Anogeissus leiocarpus et Terminalia glaucescens ainsi que les savanes herbeuses $(12,5 \%)$ à Flueggea virora et Brachiaria deflexa. Ces habitats dans $85 \%$ des cas présentent un recouvrement global inférieur à $50 \%$, et des strates arbustives (65\%) et arborescentes (25\%) dominantes, caractéristique des milieux ouverts. Les sols y sont argileux. Un total de 17 points d'eau a été recensé constitués principalement de cours d'eau à $65 \%$ et de marigots (35\%). Ces sources tarissent toutes en saison sèche. Le Tableau 2 présente les fréquences des facteurs de l'habitat.

\section{Régime alimentaire des phacochères}

Le Tableau 3 présente la liste des espèces végétales consommées par les phacochères du sud Bénin ainsi que les parties consommées et les milieux de prédilection. Le Tableau 4 montre les fréquences des espèces par famille du régime alimentaire. Au total 21 espèces végétales sont reconnues comme consommées par les phacochères dans cette partie du Bénin. Ces espèces végétales sont réparties en 14 familles dont la plus importante est celle des Poaceae $(33,33 \%)$. Ces espèces sont pour la plupart savanicoles $(73,91 \%)$ et culturales $(26,09 \%)$.

Quant aux parties consommées, elles concernent les feuilles et les inflorescences de 10 espèces telles que Brachiaria deflexa, Dactyloctenium aegyptium, Eleusine indica et Panicum repens. Le tout représentant 37,04\% du nombre d'espèce de son alimentation. L'observation de diverses fibres dans les crottes de ces animaux démontre de l'importance des feuilles dans leur alimentation.

Le prélèvement des parties souterraines (racines et tubercules) se fait au niveau de 9 espèces $(33,33 \%)$. Ces plantes sont des 1910 
Curcubitaceae, des Dioscoreaceae et des Fabaceae notamment Pterocarpus erinaceus que l'animal apprécie particulièrement.

Les fruits de Nauclea latifolia, Arachis hypogea, d'Elaeis guineensis, de Vitellaria paradoxa et les grains de Zea mays dont le prélèvement est de l'ordre de $22,22 \%$ de la quantité des aliments consommée entrent également dans l'alimentation des phacochères. Ainsi que les tiges des espèces comme le Musa sp et le Saccharum officinarum.
Comme ressources animales, les phacochères consomment toute sorte d'aliment carné notamment les petits mammifères tels que les lièvres (Lepus saxatilis), les aulacodes (Thryonomys swinderianus), et les écureuils (Xerus erythropus) ainsi que les poissons, les francolins (Francolinus bicalcaratus) et leurs œufs, les serpents, les escargots, les grenouilles et les vers de terre.

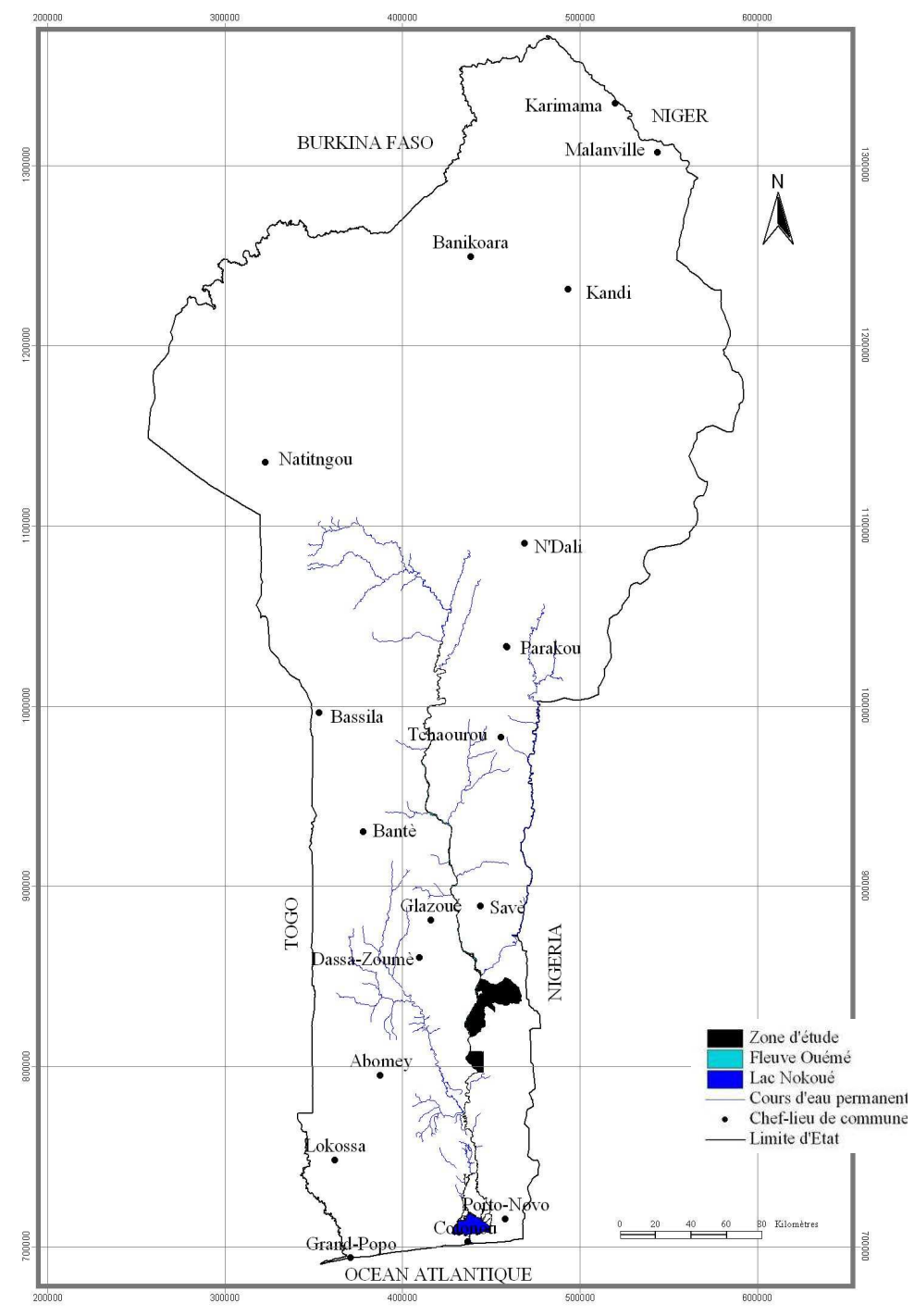

Figures 1 : Carte du Benin montrant la zone d'étude. 


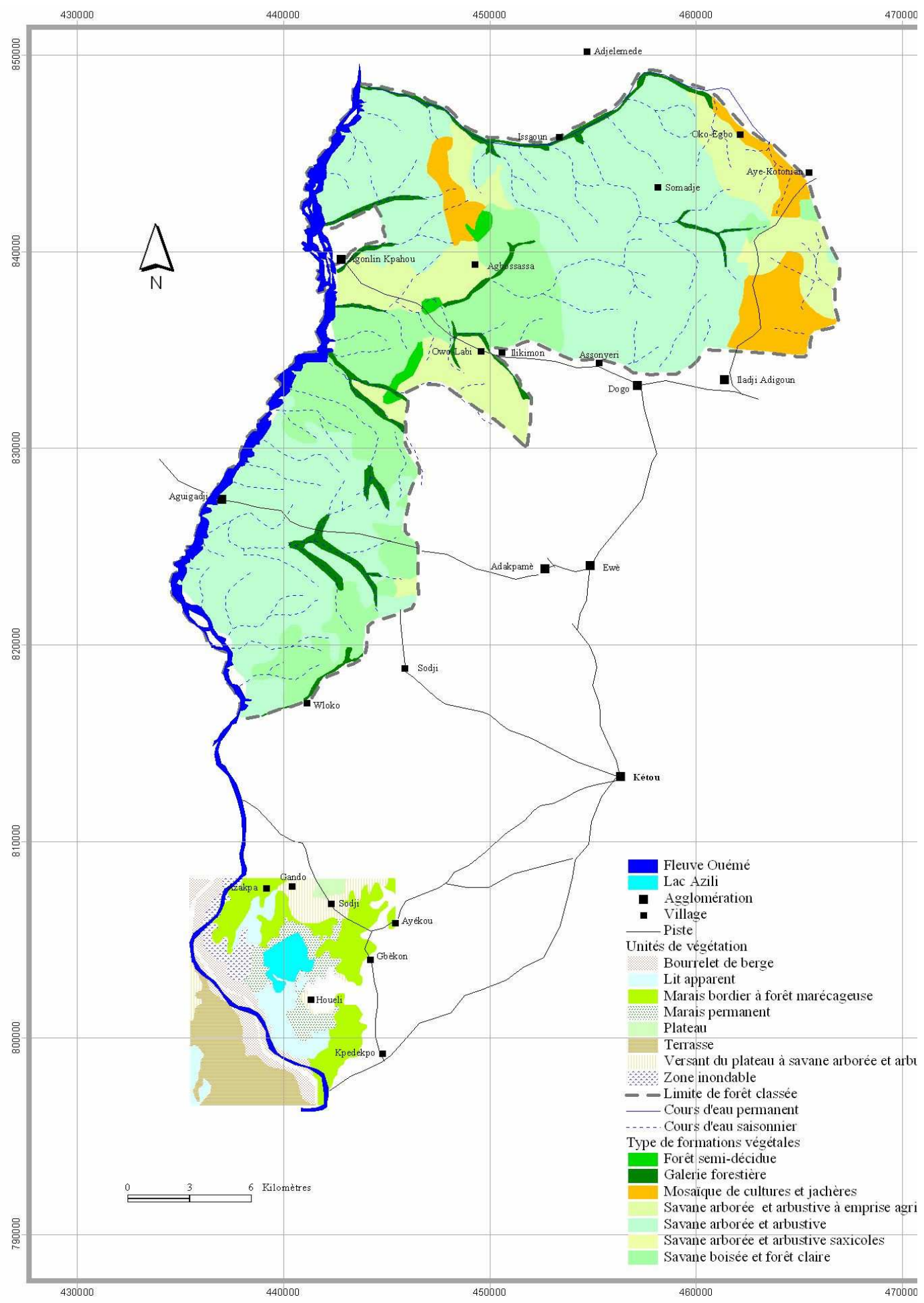

Figure 2 : Complexe forêt classée de Dogo-Kétou et forêt marécageuse du lac Azili. 


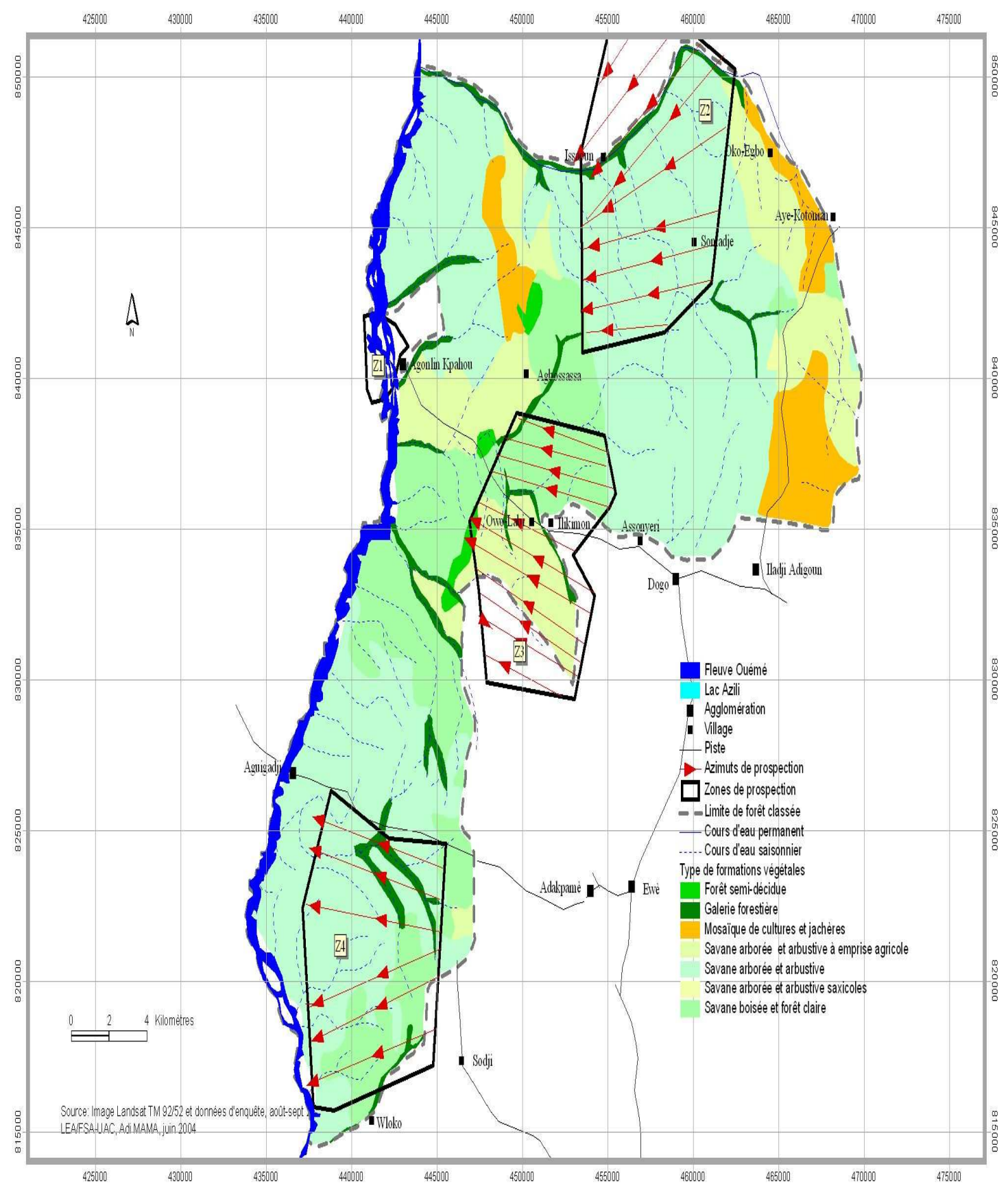

Figure 3: Localisation des aires de prospection et leurs azimuts de prospection dans la forêt classée de Dogo-Kétou. 


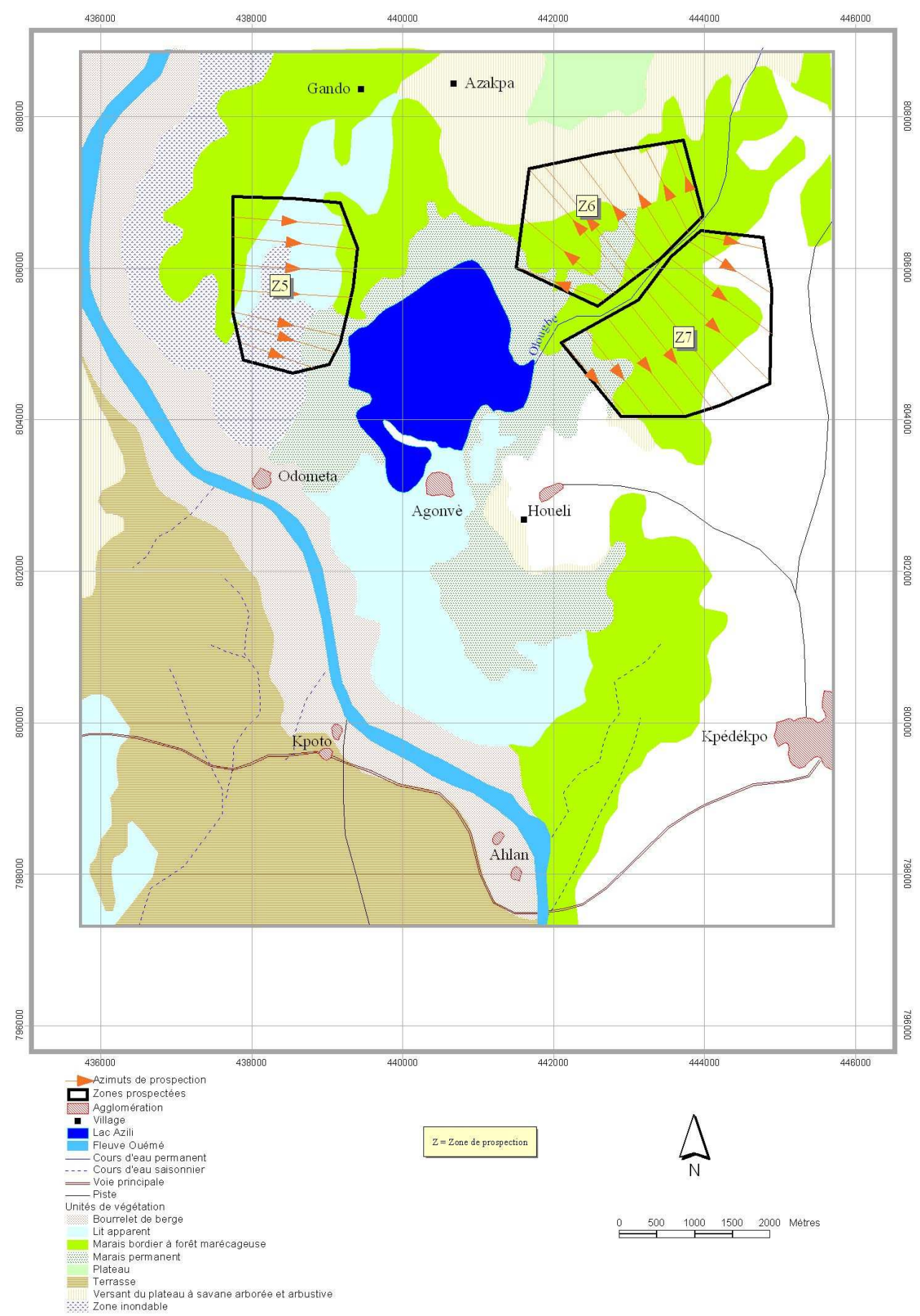

Figure 4: Localisation des aires de prospection et leurs azimuts de prospection dans la forêt marécageuse d'Azili. 


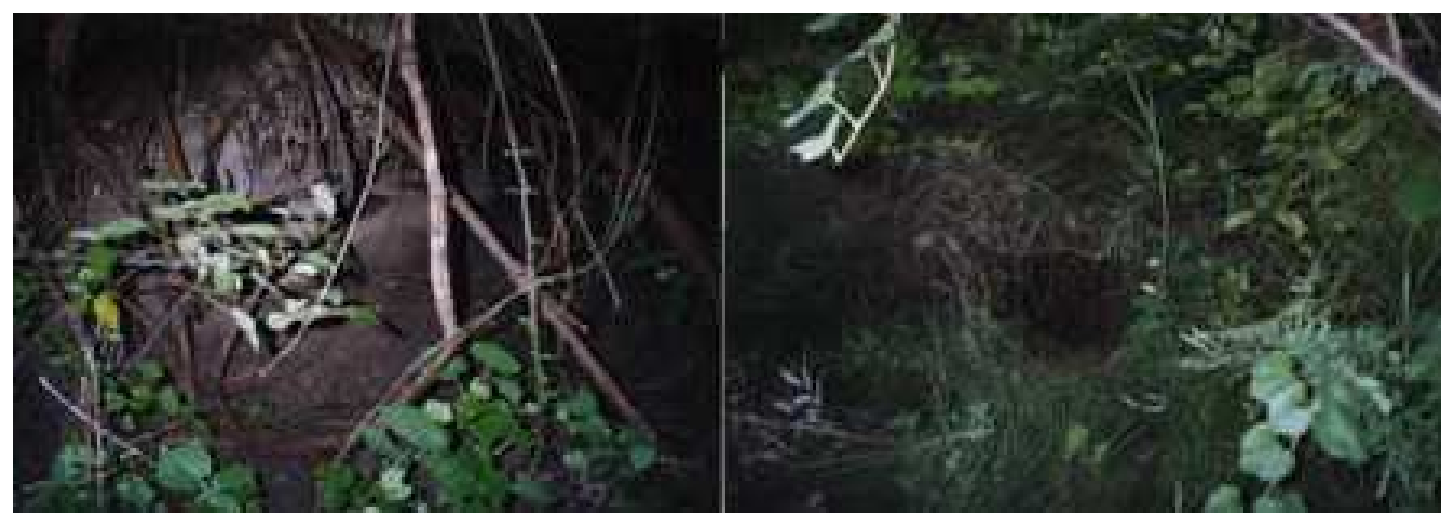

Photo1: Gîtes des phacochères (cliché ADJIN, février 2004).

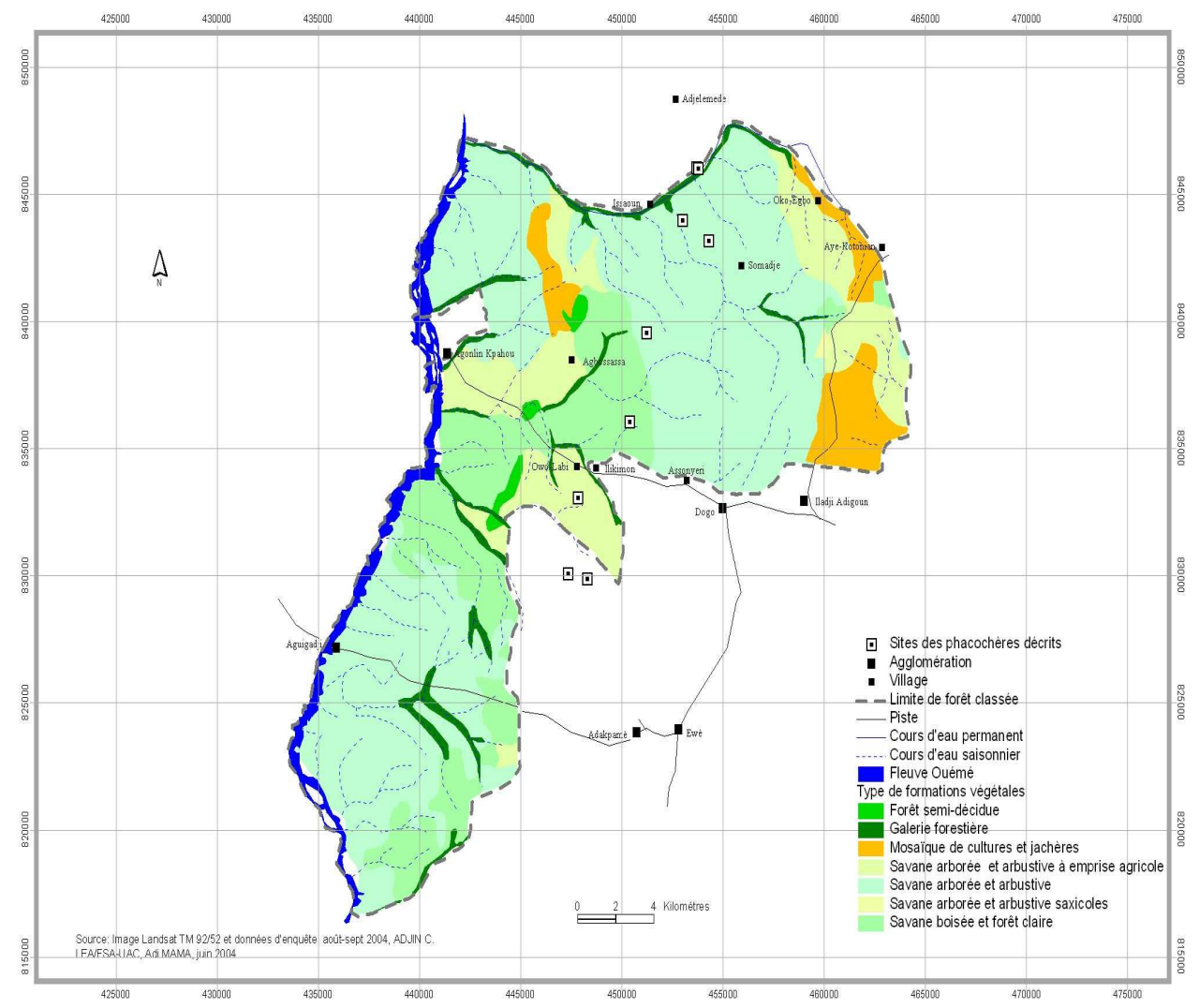

Figure 5 : Localisation des sites de phacochères décrits dans la forêt classée de Dogo-Kétou. 


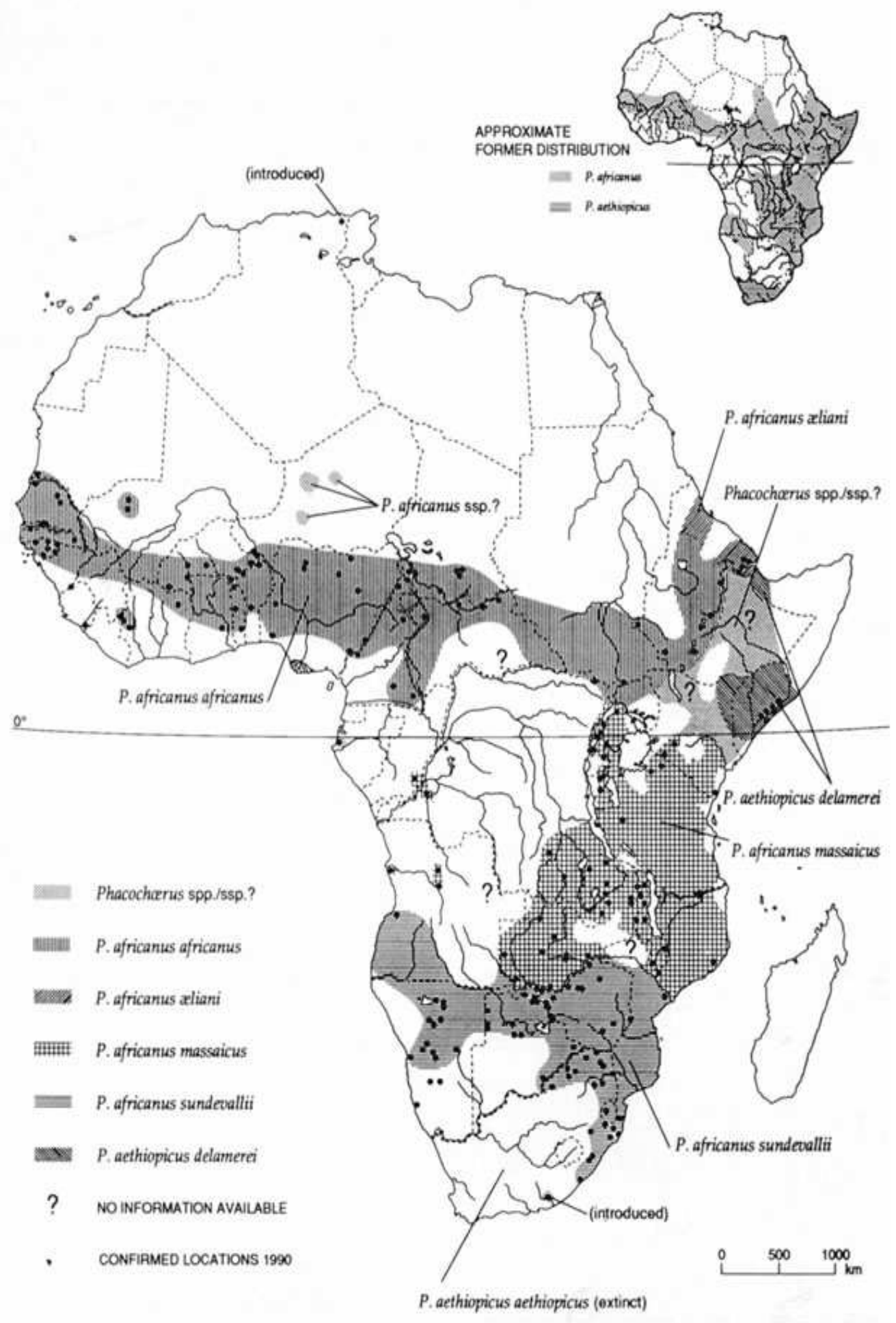

Figure 6 : Carte de distribution géographique des phacochères en Afrique d'après Vercammen et Mason (1993). 
Tableau 1 : Caractéristiques des huit gîtes de phacochères dans la forêt de Dogo-Kétou.

\begin{tabular}{lccl}
\hline Gîtes & \multicolumn{2}{c}{ Dimensions $(\mathbf{m})$} & Principales espèces végétales constitutives \\
\cline { 2 - 4 } & Hauteur & Circonférence & \\
\hline 1 & 1,2 & 20,2 & Zanthoxylum zanthoxyloides (Lam.) Watermann \\
\hline 3 & 1,8 & 22,8 & Chromolaena odorata (L.) R. M. King \& H. Robinson \\
\hline 4 & 1,8 & 9,7 & $\begin{array}{l}\text { Chromolaena odorata } \text { (L.) R. M. King \& H. Robinson } \\
\text { Anogeissus leiocapus } \text { (D.C.) Guill. \& Perr. }\end{array}$ \\
\hline 5 & 2,7 & 13,7 & $\begin{array}{l}\text { Anogeissus leiocapus } \text { (DC) Guill. \& Perr. } \\
\text { Cissus rufescens Guill. \& Perr. } \\
\text { Cissus quadrangularis L. }\end{array}$ \\
\hline 6 & 2,1 & 16,3 & $\begin{array}{l}\text { Cissus rufescens Guill. \& Perr. } \\
\text { Cissus quadrangularis L. } \\
\text { Ampelocissus bombycina Planch. }\end{array}$ \\
\hline 7 & 1,6 & 20,1 & $\begin{array}{l}\text { Chromolaena odorata } \text { (L.) R. M. King \& H. Robinson } \\
\text { Clueggea virosa } \text { (Roxb ex Willd.) Voigt. } \\
\text { Cissus populnea Guill. \& Perr. } \\
\text { Cissus rufescens Guill. \& Perr. } \\
\text { Cissus quadrangularis L. }\end{array}$ \\
\hline 8 & 2,5 & 17,2 & Chromolaena odorata (L.) R. M. King \& H. Robinson \\
\hline Moyenne & $1,95 \pm 0,49$ & $16,2 \pm 4,58$ & \\
\hline
\end{tabular}

Tableau 2: Fréquences des facteurs de l'habitat des phacochères.

\begin{tabular}{llcc}
\hline \multicolumn{1}{c}{ Facteurs de l'habitat } & Nombre de sites & Fréquences (\%) \\
\hline Formation & Forêt claire & 2 & 25 \\
végétale & Savane arborée & 0 & 0 \\
& Savane arbustive & 5 & 62,5 \\
& Savane herbeuse & 1 & 12,5 \\
& Total & 8 & 100 \\
\hline Recouvrement & $\mathbf{0 - 3 0}$ & 4 & 50 \\
$(\boldsymbol{\%})$ & $\mathbf{3 1 - 5 0}$ & 3 & 37,5 \\
& $\mathbf{5 1 - 7 0}$ & 1 & 12,5 \\
& Total & 8 & 100 \\
\hline Strate dominante & Arborescente & 2 & 25 \\
& Arbustive & 5 & 62,5 \\
& Herbacée & 1 & 12,5 \\
& Total & 8 & 100 \\
\hline Types de sols & Argileux & 3 & 37,5 \\
& Limoneux & 3 & 37,5 \\
& Sableux & 2 & 25 \\
& Hydromorphe & 0 & 0 \\
& Total & 8 & 100 \\
\hline
\end{tabular}


Tableau 3: Espèces végétales consommées par les phacochères.

\begin{tabular}{|c|c|c|c|}
\hline Familles & Noms scientifiques & $\begin{array}{l}\text { Organes } \\
\text { végétatifs } \\
\text { consommés }\end{array}$ & Ecosystèmes \\
\hline Araceae & $\begin{array}{l}\text { Stylochaeton lancifolius } \\
\text { Kotschy \& Peyr. }\end{array}$ & Racines & Savanes \\
\hline Arecaceae & Elaeis guineensis Jacq. & Fruits & Savanes \\
\hline Asteraceae & Aspilia bussei & Feuilles & Savanes \\
\hline Balanophoraceae & Thonningia sanguinea & Feuilles, Racines & Savanes \\
\hline Curcubitaceae & Momordica basalmina L. & Racines & Savanes \\
\hline Dioscoreaceae & Dioscorea alata $\mathrm{L}$. & Tubercules & Champs+jachères \\
\hline Euphorbiaceae & Manihot esculenta Crantz. & Racines & Champs+jachères \\
\hline Fabaceae & $\begin{array}{l}\text { Arachis hypogea } \mathrm{L} . \\
\text { Pterocarpus erinaceus } \\
\text { Poir. }\end{array}$ & $\begin{array}{c}\text { Feuilles, Fruits } \\
\text { Feuilles, Racines }\end{array}$ & $\begin{array}{l}\text { Champs } \\
\text { Savanes }\end{array}$ \\
\hline Musaceae & Musa sp & $\begin{array}{c}\text { Feuilles, Fruits, } \\
\text { Tiges }\end{array}$ & $\begin{array}{c}\text { Savanes, } \\
\text { Champs+jachères }\end{array}$ \\
\hline \multirow[t]{7}{*}{ Poaceae } & $\begin{array}{l}\text { Brachiaria deflexa } \\
\text { (Schumacher) CE Hubb } \\
\text { ex Robyns }\end{array}$ & Feuilles & Savanes \\
\hline & $\begin{array}{l}\text { Dactyloctenium } \\
\text { aegyptium (L.) Beauv. }\end{array}$ & $\begin{array}{c}\text { Feuilles, } \\
\text { Inflorescences }\end{array}$ & Savanes \\
\hline & $\begin{array}{l}\text { Eleusine indica (L.) } \\
\text { Gaertn. }\end{array}$ & $\begin{array}{c}\text { Feuilles, } \\
\text { Inflorescences }\end{array}$ & Savanes \\
\hline & Panicum repens $\mathrm{L}$. & Feuilles & Savanes \\
\hline & Saccharum officinarum L. & Tiges & $\begin{array}{c}\text { Savanes, } \\
\text { Champs+jachères }\end{array}$ \\
\hline & Setaria barbata Kunth. & Feuilles, Racines & Savanes \\
\hline & Zea mays L. & Grains & Champs+jachères \\
\hline Rubiaceae & $\begin{array}{l}\text { Nauclea latifolia Smith } \\
\text { Bruce }\end{array}$ & Fruits & Savanes \\
\hline Sapotaceae & $\begin{array}{l}\text { Vitellaria paradoxa } \\
\text { Gaertn. F. }\end{array}$ & Fruits & Savanes \\
\hline Vitaceae & $\begin{array}{l}\text { Cissus populnea Guill. \& } \\
\text { Perr. }\end{array}$ & Racines & Savanes \\
\hline Zingiberaceae & $\begin{array}{l}\text { Siphonochilus aethiopicus } \\
\text { (Schweif.) B. L. Burt }\end{array}$ & Racines & Savanes \\
\hline
\end{tabular}


Tableau 4 : Fréquences des espèces par familles du régime alimentaire.

\begin{tabular}{lcc}
\hline Familles & $\begin{array}{c}\text { Nombre } \\
\text { d'espèces }\end{array}$ & Fréquences (\%) \\
\hline Araceae & 1 & 4,76 \\
Arecaceae & 1 & 4,76 \\
Asteraceae & 1 & 4,76 \\
Balanophoraceae & 1 & 4,76 \\
Curcubitaceae & 1 & 4,76 \\
Dioscoraceae & 1 & 4,76 \\
Euphorbiaceae & 1 & 4,76 \\
Fabaceae & 2 & 9,52 \\
Musaceae & 1 & 4,76 \\
Poaceae & 7 & 33,33 \\
Rubiaceae & 1 & 4,76 \\
Sapotaceae & 1 & 4,76 \\
Vitaceae & 1 & 4,76 \\
Zingiberaceae & 1 & 4,76 \\
Total & $\mathbf{2 1}$ & 100 \\
\hline
\end{tabular}

\section{DISCUSSION}

\section{Habitat des phacochères}

Cette étude met en évidence la présence effective du phacochère commun dans le Bénin méridional, dans une région phytogéographique à affinités guinéocongolaises. L'aire d'occupation de l'espèce est comprise entre les latitudes $7^{\circ} 14^{\prime}$ et $7^{\circ} 40^{\prime}$ $\mathrm{N}$. Aucun travail sur la faune n'avait encore mentionné l'existence de l'espèce à cette latitude au Bénin. Les inventaires de Sinsin et al. (1998) révélaient que l'espèce se retrouve jusque dans les massifs forestiers des Monts Kouffé. En se référant aux cartes de distribution établies par D'Huart (1991) et Vercammen et Mason (1993) (Figure 7), la partie méridionale béninoise n'est pas prise en compte.

Avec cette étude, l'aire de distribution du phacochère au Bénin s'élargit ainsi un peu plus au sud. Sa présence dans une zone guinéo-congolaise, remet en partie en cause certaines considérations écologiques faites sur ces Suidés. La présence du phacochère pourrait pourtant trouver des explications dans les influences climatiques de types soudano- guinéennes que subit la région de Kétou depuis des décennies, et le Dahomey Gap qui occasionne une incursion de savanes dans une zone qui devrait être forestière. Ajouté à cela, il y a la pression des activités anthropiques qui occasionnent la dégradation des formations forestières ; ce qui permet une avancée de la savane d'où une accommodation des taxons de savane comme c'est le cas pour le phacochère.

Cette distribution observée est peut être également due à la saisonnalité. En effet, l'étude étant faite pendant la saison des pluies, l'importance des mares et cours d'eau temporaires aurait regroupé les phacochères. Dès lors que toutes les mares se seraient asséchées, les animaux se retrouveraient donc plutôt au niveau des sources d'eau permanentes à savoir le fleuve Ouémé et la rivière Issaoun élargissant l'aire de distribution. De telles migrations saisonnières ressembleraient à celles observées par Cumming (1975) et Mason (1982), dans la zone du Sahel et ceci à cause de la rareté de l'eau dans le milieu. 
L'habitat des Suidés est principalement caractérisé par des milieux ouverts à savoir les savanes arbustives. Cependant, on les rencontre également dans des milieux fermés qui ne sont pas typiques de l'espèce notamment les savanes boisées à Anogeissus et Vitellaria qui sont similaires à des habitats décrits par Allo (1994) pour les phacochères du Parc National de Kalamoue au Cameroun.

Les proportions de marigots et de cours d'eau observées suggèrent une dépendance des phacochères par rapport à la présence de l'eau. Les marigots leur servent de bauges dans lesquelles ils se vautrent tandis que les cours d'eau assurent l'alimentation en eau. Ce sont les raisons pour lesquelles, d'Huart (1991) parle de facteur limitant à propos de l'eau.

Les phacochères de Dogo-Kétou ne dorment pas dans des terriers, mais dans des gîtes faits de buissons et de lianes entremêlées. La même observation a été faite par d'Huart et Grubb (2001) qui note que les phacochères passent la nuit sous des fourrées de couvert dense. Ces gîtes ont des fonctions similaires à celles des terriers comme la protection contre le froid et les prédateurs. Aussi, ils présentent des entrées uniques comme les terriers décrits par Miade (1998), contrairement à ceux observés par Allo (1994) dans le Kalamoue National Park (KNP) qui ont parfois des entrées multiples.

\section{Régime alimentaire}

Les phacochères de Dogo-Kétou sont omnivores. Ils consomment principalement les herbes, les feuilles, les racines et les tubercules. Mais le nombre d'espèces prélevées dans la fraction végétale est comparativement limité. En effet, les 21 espèces recensées se rapprochent des 18 espèces récoltées par Miade (1998) dans la Réserve de Biosphère de la Pendjari, mais, loin des quarante obtenues par Cumming (1975) et des 52 identifiées par Agassounon (2005) dans la forêt des Monts Kouffé. La forte proportion de graminées notée dans l'alimentation des phacochères et l'importance des fibres relevées dans leurs déjections fécales supposent également que les phacochères ont une tendance herbivore voire phytophage. De même, la nécrophagie n'est pas inhabituelle chez les phacochères du sud Bénin. Ces résultats sont en conformité avec ceux de d'Huart (1991).

\section{Conclusion}

Cette étude a permis de montrer que le phacochère commun est bien présent dans la partie sud du Bénin et plus précisément dans la forêt classée de Dogo-Kétou. Le présent travail ne représente qu'un préliminaire sur les phacochères dans le sud du Bénin. Les études complémentaires sont nécessaires afin d'approfondir les éléments majeurs qui soustendent leur présence à une telle latitude. Ces études peuvent porter sur les mensurations crâniennes des phacochères au niveau national, ce qui permettrait de déterminer les variations au sein de l'espèce au Bénin et à quelle sous-espèce appartient le phacochère du Sud-Bénin. Les hypothèses de telles recherches auraient pour bases la présence de sous-espèces non identifiées au Niger ainsi que le phénomène de migration des phacochères; l'extrême Nord du Bénin pourrait être une zone de contact entre deux sous-espèces.

\section{REMERCIEMENTS}

Nous exprimons nos remerciements aux populations locales des villages riverains des forêts de Dogo Kétou et du lac Azili pour leurs contributions à la réalisation de ce travail.

\section{REFERENCES}

Agassonon MD. 2005. Ecologie alimentaire du phacochère (Phacochoerus africanus, Gmelin 1788) dans la forêt classée de Wari-Maro au Bénin. Mémoire de 3è cycle DESS/FSA/UAC, Abomey-Calavi, $85 \mathrm{p}$.

Allo A. 1994. Ecology of the warthog Phacochoerus aethiopicus (Pallas) in Kalamoue National Park, Cameroon. UMI Dissertation Services, Michigan.

Bossou B. 2001. Contribution à 
l'aménagement de la forêt classée de Dogo-Kétou : Structure et dynamique des différents groupements végétaux et périodicité de coupes. Mémoire de 3è cycle DESS/FSA/UAC, Abomey-Calavi, $141 \mathrm{p}$.

Codjia JTC, Ekué MR, Condé SK. 2007. L'Habitat du Phacochère dans la Forêt Classée des Trois Rivières au Benin. Dans Quelles aires Protégées pour l'Afrique de l'Ouest? Conservation de la Biodiversité et Développement. éditions colloques et séminaires, IRD: Paris; 238246.

Cumming DHM. 1975. A Field study of the ecology and behaviour of warthog. Museum Memoir 7, Trustees of the National Museums and Monuments of Rhodesia, Salisbury, 175-179.

De La Fuente FR. 1979. La Faune: L'Afrique (Tome 1). Alpha Editions S.A : Paris ; 136-141.

D'Huart JP, 1991.Habitat utilisation of old world pigs. In Biologie des Suidés,. Spitz F et Barret RG (Eds). IRGM: 30-48.

D'Huart JP, Grubb P. 2001. Distribution of the common warthog (Phacochoerus africanus) and the desert warthog (Phacochoerus aethiopicus) in the Horn of Africa. African Journal of Ecology, 39(2): 156-169.

Grubb P. 1993. Taxonomy and Description. In Pigs, Peccaries and Hypos Status, Survey and Action Plan. IUCN: Cambridge; 6675.

Mason DR. 1982. Studies on the iology and ecology of warthog Phacochoerus aethiopicus in Zululand. Ph.D. Dissertation, University of Pretoria, South Africa.

Miade A. 1998. Ecologie alimentaire des phacochères (Phacochoerus aethiopicus :
Suidae) dans le Parc National de la Pendjari. Mémoire de DIT/CPU/UNB, Abomey-Calavi, $54 \mathrm{p}$.

Oliver WLR. 1995. Taxonomy and Conservation Status Of The Suiforms-An Overview. IBEX J. M. E. 3 p,

Sinsin B, Daouda I, Ahokpè E, Tèhou A, Toko I. Coubéou P, Fandohan S. 1997a. Faune sauvage des Forêts Classées de Goun-Goun, de la Sota et des Environs. Projet UNSO/BEN/LEA/FSA/UNB, Abomey-Calavi.

Sinsin B, Daoudou IE, Ahokpè Tèhou A, Toko I, Coubéou P. 1997b. Dénombrement de la faune dans les Forêts Classées de Goun-Goun, de la Sota. Document Synthèse. Projet UNSO/BEN/LEA/FSA/UNB, Ab-Calavi, Bénin, 9p.

Sinsin B, Daoudou I, Ahopkè E. 1998. Abondance et évolution des populations de mammifères des formations boisées de la région des Monts Kouffé au Bénin. Cahiers d'Ethologie, 18(2): 261-281.

Tohinlo PJY. 2001. Durabilité des Modes d'Exploitation des Ressources Naturelles en Milieu Fluvio-lacustre: Cas de Agonvè dans la Sous-Préfecture de Zagnanado. Th. d'Ing. Agr. FSA/UAC, Ab-Calavi, Bénin, 123 p.

Vercammen P, Mason DR. 1993. The warthogs (Phacochoerus africanus and $P$. aethiopicus). In Pigs, Peccaries and Hippos Status, Survey and Action Plan. IUCN: Cambridge. [En ligne]http://www. iucn.org/themes/ssc/sgs/pphsg/Apchap42.htm 03/11/2004. 